\title{
Analysis of Oversulfation in a Chondroitin Sulfate Oligosaccharide Fraction from Bovine Aorta by Nanoelectrospray Ionization Quadrupole Time-of-Flight and Fourier-Transform Ion Cyclotron Resonance Mass Spectrometry
}

\author{
Michael Mormann* and Alina D. Zamfir* \\ Institute for Medical Physics and Biophysics, Biomedical Analysis Department, University of Münster, \\ Münster, Germany \\ Daniela G. Seidler and Hans Kresse \\ Institute of Physiological Chemistry and Pathobiochemistry, University of Münster, Münster, Germany
}

Jasna Peter-Katalinić

Institute for Medical Physics and Biophysics, Biomedical Analysis Department, University of Münster, Münster, Germany

\begin{abstract}
A combination of negative ion nano-electrospray ionization Fourier-transform ion cyclotron resonance and quadrupole time-of-flight mass spectrometry was applied to analysis of oversulfation in glycosaminoglycan oligosaccharides of the chondroitin sulfate type from bovine aorta. Taking advantage of the high-resolution and high mass accuracy provided by the FT-ICR instrument, a direct compositional assignment of all species present in the mixture can be obtained. An oligosaccharide fraction containing mainly hexasaccharides exhibited different levels of sulfation, indicated by the presence of species with regular sulfation pattern as well as oversulfated oligosaccharides with one additional sulfate group. Oversulfation can be directly identified from the high-resolution/high mass accuracy FT-ICR mass spectra according to their specific isotopic fine structure. Location of sulfate groups was analyzed by Q-TOF MS and low-energy CID MS/MS. Tetrasulfated hexasaccharides were analyzed by use of collision-induced dissociation at variable collision energy for an unambiguous assignment of the attachment site of the sulfate groups by minimizing unspecific neutral losses. Cleavage of glycosidic bonds gave rise to B- and C-type ions and their respective complementary Y-and Z-type fragment ions. (J Am Soc Mass Spectrom 2007, 18, 179-187) (c 2007 American Society for Mass Spectrometry
\end{abstract}

$\mathrm{P}$ roteoglycans (PGs) are ubiquitously distributed in connective tissue and on the cell surface of mammalian tissues. They are actively participating in cell growth, differentiation and morphogenesis [1-3]. PGs consist of a core protein to which glycosaminoglycan (GAG) chains are attached through a specific oligosaccharide linkage region. The major biological importance of GAGs derives especially from their recognition function as GAGs have been shown to possess active domains, which interact with a number of

Published online November 7, 2006

Address reprint requests to Dr. J. Peter-Katalinić, Institute for Medical Physics and Biophysics, Biomedical Analysis Department, University of Münster, Robert Koch Strasse 31, D-48149, Münster, Germany. E-mail: jkp@uni-muenster.de

* Equal contribution from both authors. growth factors, cell membrane receptors, or other important functional proteins [4-8]. The polysaccharide structure of GAGs consists of alternating blocks with characteristic epimerization and sulfation patterns. With respect to the structural type of the disaccharide repeating unit, GAG chains are categorized into chondroitin sulfate (CS), dermatan sulfate (DS), heparan sulfate (HS), heparin, hyaluronic acid (HA), and keratan sulfate (KS) DS, previously called chondroitin sulfate B, and CS are structurally similar. In CS, the hexuronic acid within the repeating disaccharide unit is the D-glucuronic acid (GlcA), whereas that in DS is either L-iduronic (IdoA) or D-glucuronic acid. In DS two types of disaccharide units are found, viz., -4GlcA $\beta 1$ 3 GalNAc $\beta 1$ - and -4IdoA $\alpha 1-3$ GalNAc $\beta 1$-. The standard disaccharide unit is modified by sulfation in the Gal- 
NAc moiety. The GalNAc unit may be sulfated in position 4 or 6 , while a minor proportion of the uronic acid may be sulfated in position 2, if an additional sulfate is present. The GlcA residues are found in disaccharides containing either GalNAc-4-O-sulfate, GalNAc-6-O-sulfate, or GalNAc, while the IdoA is exclusively attached to the GalNAc-4-O-sulfate [9]. The modification of IdoA by sulfation gives rise to an increased charge density within the GAG chain being one factor controlling the binding affinity and determining the functional interaction with potential binding partners [9].

In view of the relevance of GAG oligosaccharides in many biological systems, systematic studies were conducted for the complete compositional and structural characterization on one hand and for correlating the GAG saccharide sequence with their complex functions on the other [10-15]. There is an emerging evidence that the function of CS/DS GAGs is affected by their diverse assembly with respect to chain length, variable sulfation, and IdoA contents [9]. Hence, the development of specific, sensitive, and accurate methods for their detailed structural analysis is required.

In the past decade, mass spectrometric investigation of gaseous ions generated by either matrix assisted desorption/ionization (MALDI) or electrospray ionization (ESI) contributed an essential progress to the field $[10-13,15-18]$. The most severe problems in the analysis of GAG mixtures were found to be associated to the ionization of intact long GAG chains, in-source fragmentation of the labile sulfate groups, generation of the multiply charged ions of high molecular weight GAG species of reduced sulfate content, and finally to the impossibility to distinguish ions of close $\mathrm{m} / \mathrm{z}$ values [19-21]. Moreover, in the case of ESI MS, the spectra often exhibit complex pattern even for a single component, since ions bearing different charge states and, in many cases, one or more alkali counter ions, give rise to signals at different $\mathrm{m} / \mathrm{z}$ values. For complex mixtures, the spectrum might, therefore, become impossible to interpret due to the overlapping of isobaric species. For these reasons, the structural analysis of complex GAG mixtures claimed lately for the combination of MS with efficient separation techniques such as capillary electrophoresis [19, 20, 22, 23], high-performance liquid chromatography $[13,24]$, high-performance cation exchange [25], or size exclusion chromatography [26]. These strategies, however, include several analytical steps such as separation, MS screening, and sequencing of the GAG species in on-line, on-the-fly tandem MS [10], entailing specially designed interfaces and laborious optimization procedures.

Fourier-transform ion cyclotron resonance mass spectrometry (FT-ICR MS) represents an alternative approach for the analysis of complex mixtures owing to its unique specifications such as high resolving power, mass accuracy, high sensitivity, and dynamic range [27]. Several studies have demonstrated the potential of FT-ICR MS for complex mixture elucidation, e.g., crude oil samples [28], peptide mixtures from digestion procedures [29], or glycoconjugate mixtures [30]. McClellan et al. have studied the fragmentation of regularly sulfated CS-oligosaccharides by use of SORI-CID FT-ICR MS [16] and Aguilan et al. demonstrated the potential of Nano-ESI FT-ICR MS for the analysis of $\alpha$-carrageenan sulfated oligosaccharides [31]. Recently, the noncovalent binding of chemokines to heparin octasaccharides and other potential ligands has been examined by use of high resolution FT-MS techniques [4, 5, 32].

In the present study, we describe the potential of FT-ICR MS to detect and analyze regularly sulfated and oversulfated GAG species in a mixture of oligosaccharides obtained from bovine aorta taking advantage of the high resolving power and accurate mass determination. Furthermore, nano-ESI Q-TOF CID performed at different collision energies provides information for the unambiguous determination of the sulfation position.

\section{Experimental}

\section{Reagents and Materials}

Methanol was obtained from Merck (Darmstadt, Germany) and used without further purification. Distilled and deionized water was obtained from Mili-Q Millipore Water Systems (Bedford, MA). Aqueous sample solutions were dried in a Speed Vac SPD 111V evaporator (Savant, Düsseldorf, Germany). Omega glass capillaries used in nano-ESI experiments were purchased from Hilgenberg (Malsfeld, Germany), and in-house pulled using a vertical pipette puller model 720 (David Kopf Instruments, Tujunga, CA).

\section{Sample Preparation}

The GAG chains from hybrid CS/DS proteoglycans were obtained from bovine aorta as described previously $[1,11] .{ }^{\circ}$ Briefly, $^{\circ}$ the ${ }^{\circ} \mathrm{GAG}^{\circ}$ chains $^{\circ}{ }^{\text {were }}{ }^{\circ}$ released from the CS/DS proteoglycans $(100 \mu \mathrm{g})$ by a $\beta$ elimination reaction in $40 \mu \mathrm{L} 0.15 \mathrm{M} \mathrm{NaOH}$ and $1 \mathrm{M}$ $\mathrm{NaBH}_{4}$ for $20 \mathrm{~h}$ at $37^{\circ} \mathrm{C}$. The mixture was neutralized with $50 \%$ acetic acid, diluted with $200 \mu \mathrm{L} 150 \mathrm{mM}$ $\mathrm{NaCl}, 20 \mathrm{mM}$ Tris- $\mathrm{HCl}, \mathrm{pH}$ 7.4, and applied to a Biogel TSK DEAE-5PW (Biorad, München, Germany) anionexchange chromatography column. After washing with $1.5 \mathrm{~mL} 150 \mathrm{mM} \mathrm{NaCl}, 20 \mathrm{mM}$ Tris- $\mathrm{HCl}, \mathrm{pH}$ 7.4, free GAG chains were eluted with $1.5 \mathrm{~mL}$ of $1.0 \mathrm{M} \mathrm{NaCl}, 20$ $\mathrm{mM}$ Tris- $\mathrm{HCl}, \mathrm{pH}$ 7.4, dialyzed against water, and lyophilized. Depolymerization of CS/DS was carried out by digesting $0.5 \mathrm{mg}$ of GAGs with $1 \mathrm{mU}$ chondroitin B lyase (Seikagaku Kogyo, Tokyo, Japan) in $200 \mu \mathrm{L} 50$ $\mathrm{mM}$ Tris/ $\mathrm{HCl}, \mathrm{pH}$ 8.0, containing $60 \mathrm{mM}$ sodium acetate, $60 \mathrm{mM} \mathrm{NaCl}, 0.01 \%$ bovine serum albumin and $3 \mathrm{mM} \mathrm{NaN}$ for $2 \mathrm{~h}$ at $37^{\circ} \mathrm{C}$. After the first hour of incubation, $1 \mathrm{mU}$ of enzyme was added again. The enzyme cleaves the linkages in DS between GalNAc and L-IdoA by elimination irrespective of the sulfation 
pattern. Size fractionation of oligosaccharides was performed on a Superdex Peptide HR10/30 column (Amersham-Pharmacia, Freiburg, Germany) equilibrated and eluted with $0.5 \mathrm{M} \mathrm{NH}_{4} \mathrm{HCO}_{3}$ at a flow rate of $0.5 \mathrm{~mL} / \mathrm{min}$ with $\mathrm{UV}$ detection at $232 \mathrm{~nm}$. Fractions of $150 \mu \mathrm{L}$ were collected, and the hexasaccharide peak of CS was pooled and desalted on a prepacked D-Salt column, MW 1000 (Perbio Science, Bonn, Germany).

\section{Mass Spectrometry}

Nanoelectrospray quadrupole time-of-flight tandem mass spectrometry (nano-ESI-Q-TOF-MS/MS). Nano-ESI-QTOF-MS and tandem MS was performed with an orthogonal hybrid quadrupole time-of-flight mass spectrometer (Q-TOF Micromass, Manchester, United Kingdom) equipped with a Z-spray ion source.

For nano-ESI-QTOF-MS experiments, about $30 \mu \mathrm{L}$ of aqueous hexasaccharide fraction was completely evaporated and the resulting dry substrate was dissolved in $20 \mu \mathrm{L} \mathrm{H}{ }_{2} \mathrm{O} / \mathrm{MeOH}(40 / 60)$ solution.

All mass spectra were acquired in the negative ion mode with a capillary voltage of $800 \mathrm{~V}$ and a sampling cone potential of $18 \mathrm{~V}$. The nano-ESI parameters, such as capillary and sampling cone potential, source block temperature $\left(80^{\circ} \mathrm{C}\right)$, desolvation gas pressure (nominal flow: $100 \mathrm{l} \mathrm{h}^{-1}$ ) were optimized to obtain a stable spray and to avoid the loss of sulfate groups from the ionized GAG molecules by in-source fragmentation. Tandem mass spectrometry was performed by collision-induced dissociation at variable kinetic energies as described previously ${ }^{\circ}\left[10,{ }^{\circ} 11\right]^{\circ}$ using $^{\circ} \mathrm{Ar}^{\circ}$ at $^{\circ} \mathrm{a}^{\circ}$ nominal $^{\circ}$ pressure $^{\circ}$ of $10^{-3} \mathrm{~Pa}$ as the collision gas. Briefly, in the CID experiment the acceleration voltage was gradually increased from 8 to $30 \mathrm{~V}$ during the ongoing MS/MS experiment to obtain all structural-informative product ions. The fragmentation spectrum was combined from the total ion chromatogram acquired for $20 \mathrm{~min}$ (corresponding to 600 scans) within the whole range of the employed collision energy. Consequently, in the CID mass spectra obtained at different collision energies, as many fragment ions as possible of the GAG molecule were detected.

Nanoelectrospray Fourier transform ion cyclotron resonance mass spectrometry. Measurements were performed using a Bruker Apex II FT-ICR mass spectrometer equipped with a $9.4 \mathrm{~T}$ actively shielded magnet. Gasphase ions were generated from solutions containing approximately $5 \mathrm{pmol} / \mu \mathrm{L}$ of the analyte material in water/methanol/ammonia (65/35/0.1, vol/vol/vol) by nano-ESI in the negative ion mode using an Apollo ion source. Typical source conditions were: capillary voltage: $650 \mathrm{~V}$ and a capillary exit voltage of $-60 \mathrm{~V}$ to minimize unintended capillary skimmer dissociation as well as formation sodium adducts. The electrospray generated ions were accumulated for $0.5 \mathrm{~s}$ in the hexapole located after the second skimmer of the ion source and then transferred into the ICR cell. The ions were trapped inside the Infinity ${ }^{\mathrm{TM}}$ ICR cell either by application of a "sidekick" or without "sidekick" at different ion extraction times to trap only ions of a desired $\mathrm{m} / \mathrm{z}$ range by gated trapping. The trapping voltages were set to $-0.9 \mathrm{~V}$ at both trapping electrodes. All mass spectra were acquired in the broadband mode with $1 \mathrm{M}$ word data points. The time-domain signals were zero-filled once and apodized by a quadratic sine bell function before Fourier transformation. For all spectra shown 64 scans were accumulated. Only peaks that exhibited a sufficient signal to noise ratio to observe the corresponding isotopomeric peaks were included in the assignments.

Spectra were calibrated externally by use of the commercially available HP-calibration mixture.

\section{Results and Discussion}

\section{Structural Characterization of the Hexasaccharide Species by Nanoelectrospray Quadrupole Time-of Flight Tandem Mass Spectrometry}

The negative ion mode nano-ESI-QTOF mass spectrum of the hexasaccharide fraction obtained in the negative ion $^{\circ}$ mode $^{\circ}$ is $^{\circ}$ depicted $^{\circ}$ in $^{\circ}$ Figure $^{\circ} 1$.

The most abundant ionic species is a regularly sulfated hexasaccharide containing one $\mathrm{SO}_{3}$ per disaccharide unit: $\Delta 4,5-$ UAGalNAc[GlcAGalNAc] $_{2}(3 \mathrm{~S})$, where $\mathrm{nS}$ represents the number of the sulfate groups present in the oligosaccharide chain. This species was detected as 2-fold charged [M - 2H] $]^{2-}$ ions at $m / z$ 696.31, 3-fold charged $[\mathrm{M}-3 \mathrm{H}]^{3-}$ ions at $m / z 458.19$, 4-fold charged $[\mathrm{M}-4 \mathrm{H}]^{4-}$ ions at $m / z$ 343.28. and 5 -fold charged [M $5 \mathrm{H}]^{5-}$ ions at $m / z 274.44$.

As expected, the triply charged trisulfated hexasaccharide ions with $\mathrm{m} / \mathrm{z} 458.19$ give rise to the most intense signal for which the number of the negative charges equals the number of the sulfate groups. In the hexasaccharide molecule, a double-bond indicating the presence of the $\Delta-4,5$-UA building block was formed by elimination during the enzymatic cleavage of the polysaccharide chain (DS) by chondroitin B lyase.

Besides this trisulfated hexasaccharide, two additional hexasaccharide species, both oversulfated, were detectable. The triply charged ions at $m / z 490.77$ were assigned to a saturated tetrasulfated hexasaccharide, accompanied by triply charged ions detected at $\mathrm{m} / \mathrm{z}$ 484.72 attributed to an unsaturated tetrasulfated hexasaccharide, indicating the presence of the $\Delta-4,5-\mathrm{UA}$ at the nonreducing end like in $\Delta$-4,5-UA GalNAc[Glc$\mathrm{AGalNAc}_{2}(4 \mathrm{~S})$. The same pattern is found for the triply charged ions at $m / z 517.31$ corresponding to the saturated hexasaccharide along with the unsaturated congener at $m / z 511.39$ assigned to the HexAGalNAc[GlcAGalNAc $_{2}(5 S)$ species.

In the pooled hexasaccharide fraction, small amounts of octasaccharide species were detected. The spectrum reveals the presence of an octasaccharide, i.e., HexAGalNAc[GlcAGalNAc] $]_{3}(4 S)$ of regular sulfation 


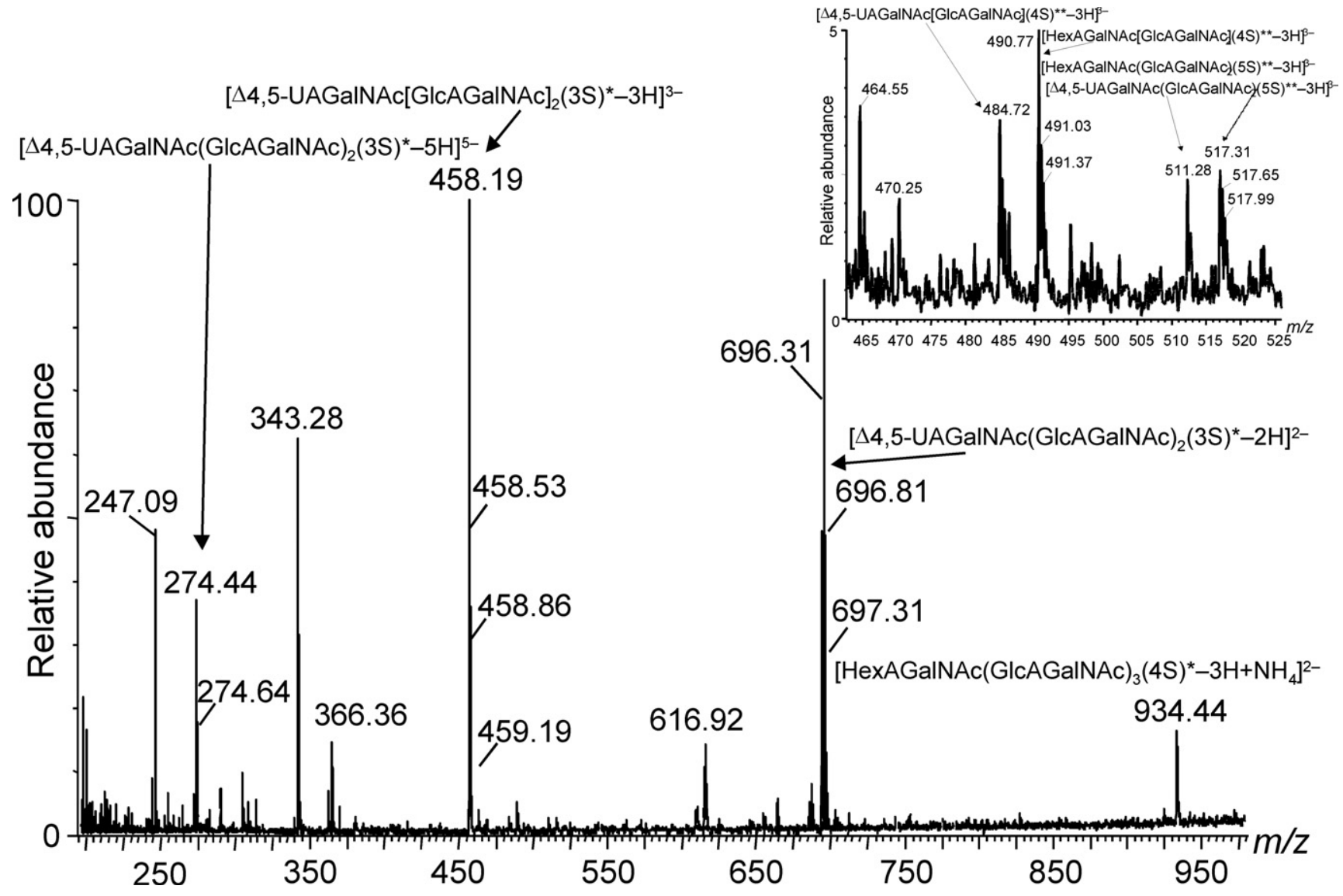

Figure 1. Negative ion mode nano-ESI-Q-TOF mass spectrum of the CS hexasaccharide fraction from bovine aorta. The inset shows a mass scale expansion of $\mathrm{m} / \mathrm{z} 458-525$. Solvent: water/methanol (40/60); ESI capillary potential $800 \mathrm{~V}$; sampling cone potential $18 \mathrm{~V}$. One asterisk denotes fully sulfated species, two asterisks denote oversulfated species.

pattern. This species has been detected as doubly charged ions of low abundance at $\mathrm{m} / \mathrm{z}$ 934.44. An interesting aspect is that the content of undersulfated hexasaccharide structures either present in the mixture and/or formed by sulfate loss by in-source decay is rather low. The disulfated hexasaccharide species was barely detectable at $\mathrm{m} / \mathrm{z} 431.24$ while the monosulfated species was not observed at all.

To further elucidate the structure of the oversulfated species present in the mixture the triply charged ions of $\mathrm{m} / z 484.72$ corresponding to a composition of $\Delta-4,5-\mathrm{UA}$ GalNAc[GlcAGalNAc] $_{2}(4 \mathrm{~S})$ hexasaccharides have been chosen for sequencing in a tandem MS experiment by applying ${ }^{\circ} \mathrm{CID}^{\circ} \mathrm{at}^{\circ}$ variable $^{\circ}$ collision $^{\circ}$ energies $^{\circ}\left(\mathrm{cf}^{\circ}{ }^{\circ}\right.$ Figure $2 \mathrm{a},{ }^{\circ} \mathrm{b},{ }^{\circ} \mathrm{c},{ }^{\circ}$ and ${ }^{\circ} \mathrm{d}$ ).

Under optimized CID conditions, a high number of diagnostic fragment ions relevant for structural elucidation was obtained. The fragment ions were assigned (cf. ${ }^{\circ}$ Figure ${ }^{\circ}$ ) ${ }^{\circ}$ assuming ${ }^{\circ}$ that ${ }^{\circ}$ chondroitin ${ }^{\circ} \mathrm{B}^{\circ}$ lyase $^{\circ}$ cleavage leads to formation of a 4,5 double-bond in the IdoA building block at the nonreducing end. The presence of fully and oversulfated fragment ions demonstrates that under CID conditions used in this study the cleavage of all glycosidic bonds was detected whereas the desulfa- tion process has been induced only to a limited extent. The rather large number of scans accumulated under extremely mild MS/MS conditions enhanced the formation of doubly charged fragment ions, reasonably more stable ${ }^{\circ}$ with $^{\circ}$ respect $^{\circ}$ to ${ }^{\circ}$ the ${ }^{\circ}$ sulfate ${ }^{\circ}$ group ${ }^{\circ}$ loss $^{\circ}[17] .^{\circ}$ The fragment ions $Y_{1}, Z_{1}, Y_{2}, Y_{3}$, and $Z_{3}$ documented a part of the ${ }^{\circ}$ sequence at ${ }^{\circ}$ the ${ }^{\circ}$ reducing ${ }^{\circ} \mathrm{end}^{\circ}$ (cf. ${ }^{\circ}$ Figure 2$)$. These ions characteristic for the regular sulfation have ascertained the distribution of the sulfate moieties at the reducing end. The nonreducing end of the molecule was characterized by $\mathrm{B}_{2}, \mathrm{C}_{2}$ (or $\left.\mathrm{Z}_{2}\right), \mathrm{B}_{3}$, and $\mathrm{C}_{3}$ fragment ions corresponding to the nonsulfated $\mathrm{UA}$ and the

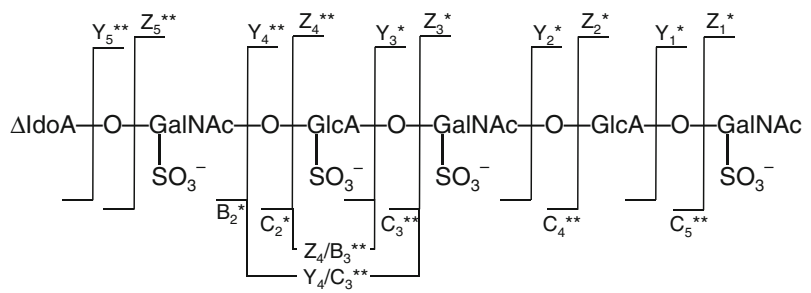

Scheme 1. Fragmentation scheme and derived structure of the tetrasulfated CS hexasaccharide from bovine aorta determined by CID-MS/MS. 

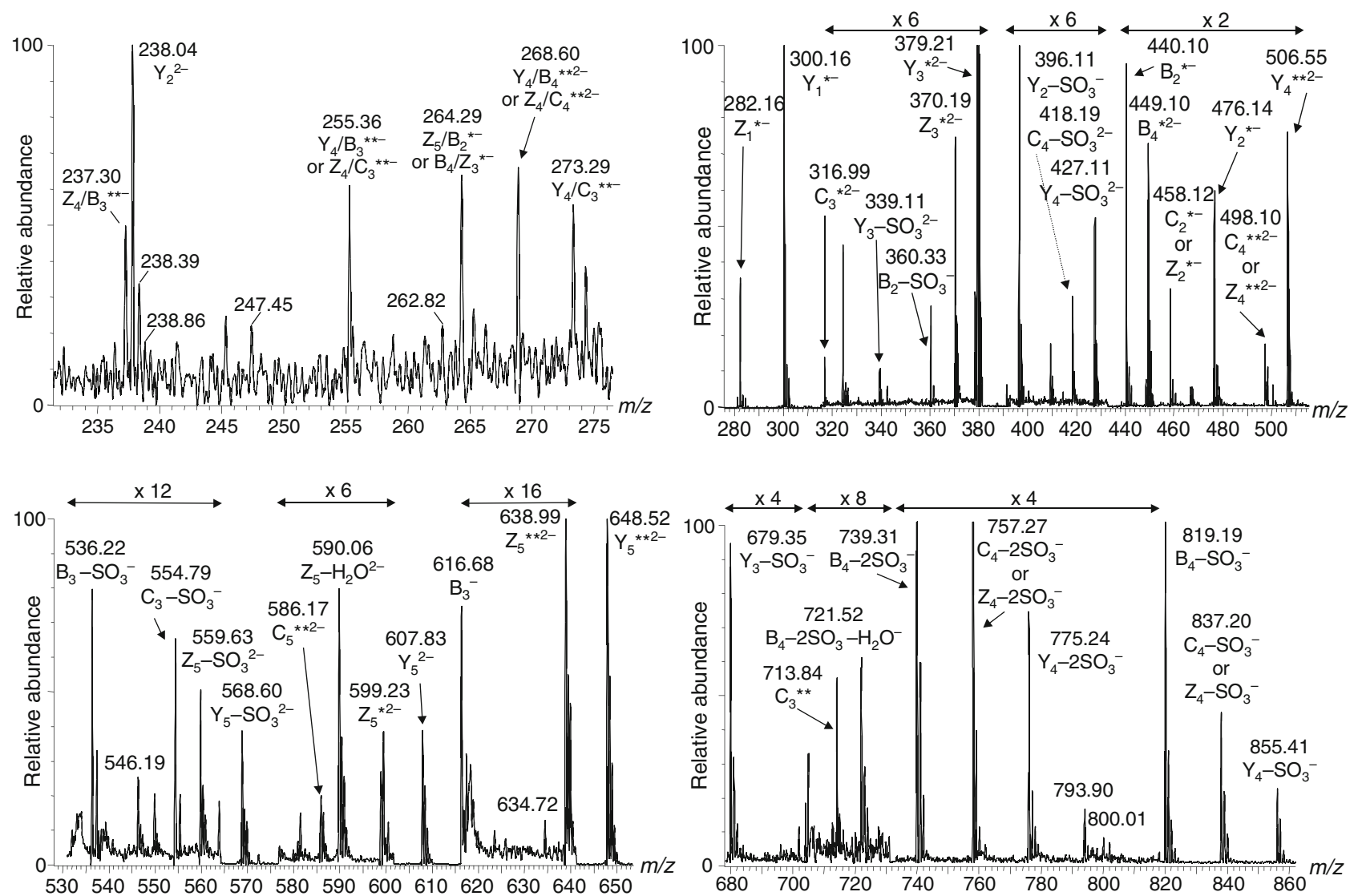

Figure 2. Negative ion mode nano-ESI-QTOF-MS/MS spectrum of the triply charged tetrasulfated CS hexasaccharide species detected at $m / z$ 484.60. (a) $m / z: 235-275$, (b) $m / z: 280-510$, (c) $m / z: 530-650$, (d) $m / z$ : 608-860. Acceleration voltage: 8-30 V. One asterisks denotes fully sulfated species, two asterisks denote oversulfated species.

monosulfated IdoAGalNAc, exhibiting clear evidence for the localization of the $\mathrm{SO}_{3}$ group at the GalNAc moiety of the disaccharide. Since $\Delta$-unsaturated CS oligosaccharide ions would lead to isobaric $C_{n}$ and $Z_{n}$ fragment ions, if $\mathrm{n}$ is an even number, no unambiguous assignment $^{\circ}$ of $^{\circ}$ these $^{\circ}$ species $^{\circ}$ is ${ }^{\circ}$ possible $^{\circ}[16] .{ }^{\circ}$ The $^{\circ}$ six oversulfated sequence ions, i.e., $\mathrm{C}_{3}{ }^{*-}, \mathrm{C}_{4}{ }^{* * 2-}$ (or $\left.\mathrm{Z}_{4}{ }^{* * 2-}\right), \mathrm{C}_{5}^{* * 2-}, \mathrm{Y}_{4}^{* * 2-}, \mathrm{Y}_{5}^{* * 2-}, \mathrm{Z}_{5}{ }^{* 2-}$; generated by cleavage of the glycosidic bonds provided evidence for the localization of the additional sulfate at the first GlcA entity from the non-reducing end in the hexasaccharide molecule ${ }^{\circ}\left(\mathrm{cf}^{\circ}{ }^{\circ}\right.$ Figure $^{\circ}{ }^{\circ}{ }^{\circ}$ and ${ }^{\circ} S$ cheme 1 ). ${ }^{\circ}$ By ${ }^{\circ}$ increasing ${ }^{\circ}$ the collision energy during the CID experiment, internal cleavages rendering shorter sequences were obtained. The latter provided useful information on the structure of the repeating disaccharide unit of the GAG chain and, in particular, for the determination of the fourth sulfate group position. The fragment ions of $m / z 237.30$ $\left(\mathrm{Z}_{4} / \mathrm{B}_{3}{ }^{*-}\right)$ and $m / z 273.29\left(\mathrm{Y}_{4} / \mathrm{C}_{3}{ }^{* *-}\right)$ are unique for a sulfated glucuronic acid at the third monosaccharide unit from the nonreducing end, therefore, corroborating the assignment of the position of the additional sulfate group. The structure of the $\Delta-4,5-\mathrm{UA}$ GalNAc$\left[\mathrm{GlcAGalNAc}_{2}(4 \mathrm{~S})\right.$ hexasaccharide, which was derived from CID experiments performed at different collision energies, is shown in Scheme $\mathbf{1}$.
Analysis of the Glycosaminoglycan Mixture by Nanoelectrospray Fourier Transform Ion Cyclotron Resonance Mass Spectrometry

To apply an alternative approach for the exploration of the sulfation status of the oligosaccharide species present high-resolution FT-ICR-MS has been considered for analysis of the gas-phase ions generated by nano-ESI from the same mixture.

A representative negative ion mode nano-ESI FT-ICR mass spectrum of the hexasaccharide fraction obtained under ${ }^{\circ}$ sidekick $^{\circ}$ trapping $^{\circ}$ conditions $^{\circ}$ is ${ }^{\circ}$ shown $^{\circ}$ in $^{\circ}$ Fig- $^{\circ}$ ure 3 .

The high resolving power of the instrument used ( $~ 80,000$ in the broadband mode at $\mathrm{m} / \mathrm{z} 1000$ ) allows the detection of more than 80 singly, doubly, and triply charged distinct ionic species (without taking into account isotopomeric species) corresponding to 15 molecular species. The mass accuracy obtained is typically better than $1.5 \mathrm{ppm}$. Almost all species detected in the Q-TOF experiment were also obtained in different charge states and different degree of cationization, i.e., mainly attachment of sodium and ammonium cations. Similar to the spectra obtained with the Q-TOF instrument, the unsaturated hexasaccharide species with reg- 


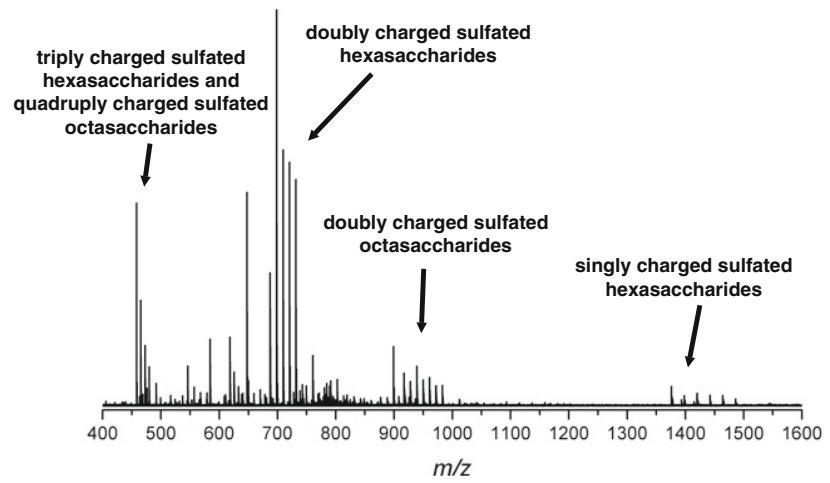

Figure 3. Negative ion nano-ESI FT-ICR mass spectrum of the CS hexasaccharide fraction from bovine aorta obtained by side-kick trapping.

ular sulfation pattern gives rise to the most intense signals in the spectrum.

Since most species detected appear in different charge states and under different degrees of cationization, much information derived from the spectrum depicted ${ }^{\circ}$ in ${ }^{\circ}$ Figure ${ }^{\circ}$ appear ${ }^{\circ}$ to be $^{\circ}$ redundant. Therefore, the same sample has been measured under different trapping conditions, i.e., gated trapping without application of a sidekick. Taking advantage of the intrinsic time-of-flight effect of the instrument used in this experiment, ions appearing only in a small mass window at low $m / z$ values have been trapped. This leads to a significant reduction of the number of ionic species present in the ICR cell, enhancing the resolution under the present experimental conditions as has been demonstrated for instruments equipped with a quadrupole allowing ${ }^{\circ}$ the ${ }^{\circ}$ preselection ${ }^{\circ}$ of $^{\circ} a^{\circ}$ desired $^{\circ}$ mass ${ }^{\circ}$ range $e^{\circ}[33]$. Furthermore, it has been shown that the intrinsic resolving power of ICR instruments is an inverse function of the ${ }^{\circ} \mathrm{m} / z$ ratio ${ }^{\circ}[27]^{\circ}{ }^{\circ}$ Thus, ${ }^{\circ}$ trapping ${ }^{\circ}$ ions ${ }^{\circ}$ in ${ }^{\circ}$ higher ${ }^{\circ}$ charge states at lower $m / z$ values gives rise to more valuable information. Recently, a similar experimental approach has been introduced by Wong and Amster to increase the ${ }^{\circ}$ dynamic ${ }^{\circ}$ range $^{\circ}$ of ${ }^{\circ}$ an $^{\circ}$ FT-ICR ${ }^{\circ}$ instrument ${ }^{\circ}[34]$.

The negative ion mode nanoESI FT-ICR mass spectrum of the hexasaccharide fraction acquired by application of the intrinsic time-of-flight effect to trap preferably highly charged species with low $m / z$ values is shown ${ }^{\circ}{ }^{\circ}{ }^{F i g u r e}{ }^{\circ} 4$. The $^{\circ}$ different $^{\circ}$ species ${ }^{\circ}$ detected ${ }^{\circ}$ in the mixture along with their predicted and experimentally obtained $^{\circ} \mathrm{m} / \mathrm{z}$ values ${ }^{\circ}$ are $^{\circ}$ listed $^{\circ}$ in $^{\circ}$ Table $^{\circ} 1 .^{\circ}$ Under $^{\circ}$ the present experimental conditions, quadruply and triply charged ions, mainly [ $\Delta-4,5-\mathrm{UA}$ GalNAc(GlcAGal$\left.\mathrm{NAc}_{2}(3 \mathrm{~S}) \quad-\quad 4 \mathrm{H}\right]^{4-}$ and $[\Delta-4,5-\mathrm{UA}$ GalNAc$\left(\text { GlcAGalNAc}_{2}(3 \mathrm{~S})-3 \mathrm{H}\right]^{3-}$ with $m / z 343.29$ and 458.06, representing regularly sulfated species were detected. Even if the source conditions were adjusted to minimize in-source fragmentation, a few fragment ions were also observed. Closer inspection of the mass spectrum in the $m / z$ region near 458 , however, reveals the presence of at least ${ }^{\circ}$ two ${ }^{\circ}$ higher ${ }^{\circ}$ homologues $^{\circ}$ (cf. inset $^{\circ}$ Figure $^{\circ} 4$ ).

The high resolving power of the instrument exhibits the superposition of regularly sulfated octa- and decasaccharides which are 4- and 5-fold charged, respectively. These can be unambiguously identified by the

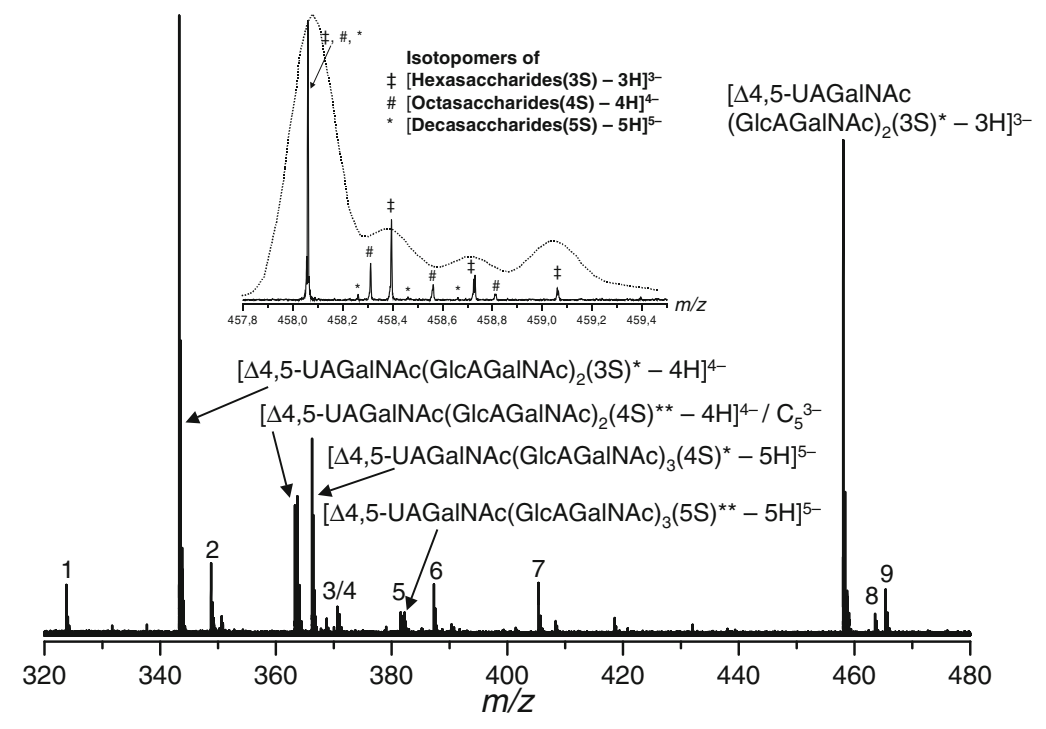

Figure 4. Negative ion nano-ESI FT-ICR mass spectrum of the CS hexasaccharide fraction from bovine aorta obtained by gated trapping. Assignment of additionally labeled ions: $1:\left[\mathrm{Y}_{5}+\mathrm{SO}_{3}-4 \mathrm{H}\right]^{4-} ; 2$ : $\left[\Delta-4,5-\mathrm{UAGalNAc}(\mathrm{GlcAGalNAc})_{2}(3 \mathrm{~S})^{*}-5 \mathrm{H}+\mathrm{Na}\right]^{4-} ; 3:\left[\Delta-4,5-\mathrm{UAGalNAc}\left(\operatorname{GlcAGalNAc}_{3}(4 \mathrm{~S})^{*}-6 \mathrm{H}+\right.\right.$ $\mathrm{Na}]^{5-} ; 4:\left[\mathrm{C}_{5}-4 \mathrm{H}+\mathrm{Na}\right]^{3-} ; 5:\left[\Delta-4,5-\mathrm{UAGalNAc}(\mathrm{GlcAGalNAc})_{4}(5 \mathrm{~S})^{*}-6 \mathrm{H}\right]^{6--} ; 6:\left[\mathrm{C}_{7}-4 \mathrm{H}\right]^{4-} ; 7:\left[\mathrm{Y}_{5}-\right.$ $3 \mathrm{H}]^{3-} ; 8:\left[\Delta-4,5-\mathrm{UAGalNAc}(\mathrm{GlcAGalNAc})_{3}(4 \mathrm{~S})^{*}-5 \mathrm{H}+\mathrm{Na}\right]^{4-} ; 9$ : $\left[\Delta-4,5-\mathrm{UAGalNAc}(\mathrm{GlcAGalNAc})_{2}(3 \mathrm{~S})^{*}\right.$ $-4 \mathrm{H}+\mathrm{Na}]^{3-}$. One asterisk denotes fully sulfated species, two asterisks denote oversulfated species. The inset shows a mass scale expansion near $\mathrm{m} / \mathrm{z} 458$ in the broadband negative nano-ESI FT-ICR mass

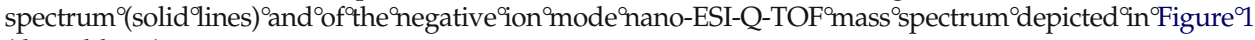
(dotted lines). 
Table 1. Theoretical and experimental $\mathrm{m} / \mathrm{z}$ values for different ionic species identified in the broadband negative nano-ESI FT-ICR mass spectrum of the hexasaccharide fraction from bovine aorta

\begin{tabular}{|c|c|c|c|}
\hline Ion & $\begin{array}{l}\text { Predicted } \\
\mathrm{m} / \mathrm{z} \text { value }\end{array}$ & $\begin{array}{l}\text { Measured } \\
\mathrm{m} / \mathrm{z} \text { value }\end{array}$ & $\begin{array}{l}\text { Error } \\
/ \mathrm{ppm}^{\mathrm{a}}\end{array}$ \\
\hline$\left[\mathrm{Y}_{5}+\mathrm{SO}_{3}-4 \mathrm{H}\right]^{4-}$ & 323.7776 & 323.7778 & +0.8 \\
\hline$\left[\Delta 4,5-\text { UAGalNAc }(\text { GlcAGalNAc })_{2}(3 S)^{*}-4 \mathrm{H}\right]^{4-}$ & 343.2939 & 343.2936 & -1.0 \\
\hline$\left[\Delta 4,5-\text { UAGalNAc }(\text { GlcAGalNAc })_{2}(3 \mathrm{~S})^{*}-5 \mathrm{H}+\mathrm{Na}\right]^{4-}$ & 348.7892 & 348.7894 & +0.7 \\
\hline$\left[\Delta 4,5-\right.$ UAGalNAc $\left(\text { GlcAGalNAc) }{ }_{2}(4 \mathrm{~S})^{* *}-4 \mathrm{H}\right]^{4-}$ & 363.2831 & 363.2832 & +0.2 \\
\hline$\left[\mathrm{C}_{5}-3 \mathrm{H}\right]^{3-}$ & 363.7156 & 363.7154 & -0.6 \\
\hline$\left[\Delta 4,5-\text { UAGalNAc }(\text { GlcAGalNAc })_{3}(4 \mathrm{~S})^{*}-5 \mathrm{H}\right]^{5-}$ & 366.2474 & 366.2471 & -0.7 \\
\hline$\left[\Delta 4,5-\mathrm{UAGaINAc}(\mathrm{GlcAGaINAc})_{3}(4 \mathrm{~S})^{*}-6 \mathrm{H}+\mathrm{Na}\right]^{5-}$ & 370.6437 & 370.6435 & -0.8 \\
\hline$\left[\mathrm{C}_{5}-4 \mathrm{H}+\mathrm{Na}\right]^{3-}$ & 371.0429 & 371.0425 & -1.1 \\
\hline$\left[\Delta 4,5-\right.$ UAGalNAc $\left(\text { GlcAGalNAc) }{ }_{4}(5 \mathrm{~S})^{*}-6 \mathrm{H}\right]^{6-}$ & 381.5496 & 381.5492 & -1.0 \\
\hline$\left[\Delta 4,5-\text { UAGalNAc }(\text { GlcAGalNAc })_{3}(5 \mathrm{~S})^{* *}-6 \mathrm{H}\right]^{6-}$ & 382.2387 & 382.2384 & -0.9 \\
\hline$\left[\mathrm{C}_{7}-4 \mathrm{H}\right]^{4-}$ & 387.3020 & 387.3016 & -0.8 \\
\hline$\left[\mathrm{Y}_{5}-3 \mathrm{H}\right]^{3-}$ & 405.3872 & 405.3869 & -0.7 \\
\hline$\left[\Delta 4,5-\text { UAGalNAc }(\text { GlcAGalNAc) })_{2}(3 \mathrm{~S})^{*}-3 \mathrm{H}\right]^{3-}$ & 458.0610 & 458.0612 & +0.4 \\
\hline$\left[{ }^{13} \mathrm{C}_{1}\right]\left[\Delta 4,5-\text { UAGalNAc }(\mathrm{GlcAGaINAc})_{4}(5 \mathrm{~S})^{*}-5 \mathrm{H}\right]^{5-}$ & 458.2617 & 458.2615 & -0.4 \\
\hline$\left[{ }^{13} \mathrm{C}_{1}\right]\left[\Delta 4,5-\text { UAGalNAc(GlcAGaINAc) }{ }_{3}(4 \mathrm{~S})^{*}-4 \mathrm{H}\right]^{4-}$ & 458.3119 & 458.3117 & -0.2 \\
\hline$\left[{ }^{13} \mathrm{C}_{1}\right]\left[\Delta 4,5-\text { UAGaINAc }(\text { GlcAGaINAc) })_{2}(3 \mathrm{~S})^{*}-3 \mathrm{H}\right]^{3-}$ & 458.3955 & 458.3951 & -0.8 \\
\hline$\left[\Delta 4,5-\text { UAGalNAc }(\text { GlcAGalNAc) })_{3}(4 \mathrm{~S})^{*}-5 \mathrm{H}+\mathrm{Na}\right]^{4-}$ & 463.5565 & 463.5565 & -0.1 \\
\hline$\left[\Delta 4,5-\text { UAGalNAc }(\text { GlcAGalNAc })_{2}(3 \mathrm{~S})^{*}-4 \mathrm{H}+\mathrm{Na}\right]^{3-}$ & 465.3883 & 465.3888 & -0.6 \\
\hline
\end{tabular}

*Fully sulfated species.

** Oversulfated species.

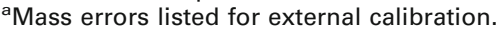

isotopic pattern found in the spectrum. The experimentally obtained $\mathrm{m} / \mathrm{z}$ values for the $\left[{ }^{13} \mathrm{C}_{1}\right]$ isotopomers of the three different homologs are in excellent agreement with $^{\circ}$ the ${ }^{\circ}$ calculated $^{\circ}$ values $^{\circ}\left(\right.$ cf. $^{\circ}$ Table $\left.^{\circ} 1\right) .^{\circ}$ Further, $^{\circ}$ the octa- and decasaccharide species also give rise to low abundant four to 6-fold charged ions. The separation of the complete isotopic pattern was not possible by using the Q-TOF instrument (resolving power of approximately 4000), therefore not allowing the detection of the higher ${ }^{\circ}$ homologues ${ }^{\circ}\left(\right.$ cf. $^{\circ}$ inset $^{\circ}$ Figure $\left.^{\circ} 4\right)$.
Besides regularly sulfated GAG species, oversulfated oligosaccharides were detected by the FT-ICR instrument as well. Ions with the formal composition of $\left.[\Delta-4,5-\mathrm{UA} \text { GalNAc(GlcAGalNAc) })_{2}(4 \mathrm{~S})-4 \mathrm{H}\right]^{4-}$ give rise to a signal at $\mathrm{m} / \mathrm{z} 363.28$ differing only by $0.2 \mathrm{ppm}$ from $^{\circ}$ the $^{\circ}$ calculated $^{\circ} \mathrm{m} / \mathrm{z}$ value ${ }^{\circ}$ (cf. $^{\circ}$ Table $^{\circ} 1$ ). ${ }^{\circ}$ Closer examination of the isotopic pattern and comparison with the respective distribution of the isotopomers of the regularly sulfated hexasaccharide confirms the presence ${ }^{\circ}$ of $^{\circ}$ an ${ }^{\circ}$ additional ${ }^{\circ}$ sulfate $^{\circ}$ group. ${ }^{\circ}{ }^{\circ}{ }^{\circ}$ Figure $^{\circ} 5,{ }^{\circ}$ mass

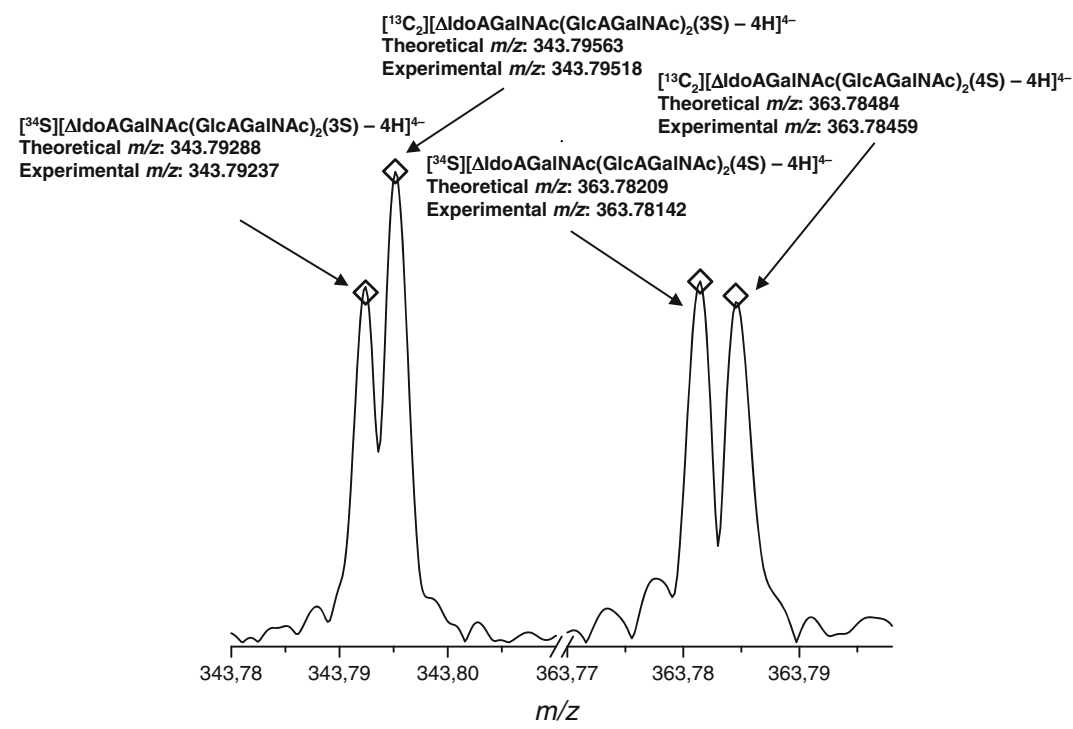

Figure 5. Mass scale expansion near $m / z 343\left(\left[\Delta-4,5-\mathrm{UAGalNAc}(\mathrm{GlcAGalNAc})_{2}(3 \mathrm{~S})^{*}-4 \mathrm{H}\right]^{4-}\right)$ and $\mathrm{m} / \mathrm{z} 363\left(\left[\Delta-4,5-\mathrm{UAGalNAc}(\mathrm{GlcAGalNAc})_{2}(4 \mathrm{~S})^{* *}-4 \mathrm{H}\right]^{4-}\right)$ in the broadband negative nanoESI FT-ICR

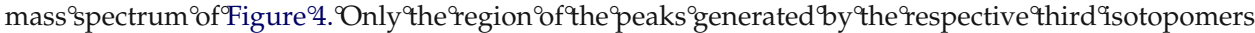
are shown. The open diamonds represent the theoretically predicted relative intensities of the signals generated by the respective isotopomers. 
scale expansions of the region for the third isotopomeric peaks together with the theoretical relative intensities at a mass resolving power of 180,000 are depicted. In both cases the peaks generated by the isotopomers containing either two 13-carbon or one 34-sulfur can be distinguished. The observed $\mathrm{m} / \mathrm{z}$ separation $(0.0028$ and $0.0031)$ are in good agreement with the theoretical separation $(0.0028)$ for the quadruply charged species under inspection. Therefore, the experimentally observed isotopic pattern for the $\Delta$-[4,5-UA GalNAc$\left(\mathrm{GlcAGalNAc}_{2}(4 \mathrm{~S})-4 \mathrm{H}\right]^{4-}$ ions clearly reflects the increased sulfur contents compared with the regularly sulfated species.

\section{Conclusions}

The analysis of an oligosaccharide oversulfation in chondroitin sulfate oligosaccharides from bovine aorta was successfully performed by use of a combination of nanoelectrospray ionization quadrupole time-of-flight (Q-TOF MS) and Fourier-transform ion cyclotron resonance mass spectrometry (FT-ICR-MS). Collision-induced dissociation of oversulfated precursor ions at various collision energies gave rise to a good sequence coverage by B- and C-type as well as $\mathrm{Y}$ - and Z-type ions formed by cleavage of the glycosidic bonds, without elimination of the relatively labile sulfate group. By this approach, a full set of sequence ions as well as diagnostic ions formed by internal cleavages were detected allowing the direct and unambiguous determination of the sulfation pattern of the species under inspection. On the other hand high-resolution/high mass-accuracy data obtained by use of FT-ICR-MS provided direct insight on the sulfation status of the oligosaccharide species present in the mixture. Using the intrinsic time-offlight effect of the instrument used in this study only ions with lower $\mathrm{m} / \mathrm{z}$ values were allowed to enter the ICR cell. By this, the number of ionic species were significantly reduced and ions with low $\mathrm{m} / \mathrm{z}$ values were trapped. Thus, the resolving power is increased without reducing the amount of information on the species present in the mixture. Resolving the isotopic fine structure and separating isotopomers containing either two ${ }^{13} \mathrm{C}$-isotopes or one ${ }^{34} \mathrm{~S}$-isotope leads directly to an identification of oversulfated species.

\section{Acknowledgments}

This work was supported by the Sonderforschungsbereich (SFB) 492 Extracellular Matrix: Biogenesis, Assembly, and Cellular Interaction (Projects Z2 and A6) of the Deutsche Forschungsgemeinschaft. Financial support by the Deutsche Forschungsgemeinschaft (DFG PE 415/14-1) and the HbfG (Land Nordrhein-Westfalen) to JPK is gratefully acknowledged. The authors thank Zygmund Budny from the Institute for Physiological Chemistry and Pathobiochemistry for help in sample preparation.

\section{References}

1. Kresse, H.; Heidel, H.; Buddecke, E. Chemical and Metabolic Heterogeneity of a Bovine Aorta Chondroitin Sulfate-Dermatan Sulfate Proteoglycan. Eur. J. Biochem.1971, 22, 557-562.

2. Taylor, K. R.; Gallo, R. L. Glycosaminoglycans and Their Proteoglycans: Host-Associated Molecular Patterns for Initiation and Modulation of Inflammation. FASEB J. 2006, 20, 9-22.

3. Kleinman, H. K.; Philp, D.; Hoffman, M. P. Role of the Extracellular Matrix in Morphogenesis. Curr. Opin. Biotechnol. 2003, 5, 526-532.

4. Yu, Y.; Sweeney, M. D.; Saad, O. M.; Crown, S. E.; Hsu, A. R.; Handel T. M.; Leary J. A. Chemokine-Glycosaminoglycan Binding: Specificity for CCR2 Ligand Binding to Highly Sulfated Oligosaccharides Using FTICR Mass Spectrometry. J. Biol. Chem. 2005, 280, 32200-32208. Erratum in: J. Biol. Chem. 2005, 280, 41124.

5. Yu, Y.; Sweeney, M. D.; Saad, O. M.; Leary, J. A. Potential Inhibitors of Chemokine Function: Analysis of Noncovalent Complexes of CC Chemokine and Small Polyanionic Molecules by ESI FT-ICR Mass Spectrometry. I. Am. Soc. Mass Spectrom. 2006, 4, 524-535.

6. Mulloy, B.The Specificity of Interactions Between Proteins and Sulfated Polysaccharides. Anais Acad. Bras. Cienc. 2005, 77, 651-664.

7. Raman, R.; Sasisekharan, V.; Sasisekharan, R. Structural Insights into Biological Roles of Protein-Glycosaminoglycan Interactions. Chem. Biol. 2005, 3, 267-277.

8. Volpi, N. Advances in Chondroitin Sulfate Analysis: Application in Physiological and Pathological States of Connective Tissue and During Pharmacological Treatment of Osteoarthritis. Curr. Pharm. Des. 2006, 12, $639-658$.

9. Trowbridge, J. M.; Gallo, R. L. Dermatan Sulfate: New Functions from an Old Glycosaminoglycan. Glycobiology 2002, 9, 117R-125R.

10. Zamfir, A.; Seidler G. D.; Kresse, H.; Peter-Katalinić, J. Structural characterization of chondroitin/dermatan sulfate oligosaccharides from bovine aorta by capillary electrophoresis and electrospray ionization quadrupole time-of-flight tandem mass spectrometry. Rapid Commun. Mass Spectrom. 2002, 16, 2015-2023.

11. Zamfir, A.; Seidler D. G.; Kresse, H.; Peter-Katalinić, J. Structural Investigation of Chondroitin/Dermatan Sulfate Oligosaccharides from Human Skin Fibroblast Decorin. Glycobiology 2003, 13, 733-742.

12. Miller, M. J.; Costello, C. E.; Malmstrom, A.; Zaia, J. A Tandem Mass Spectrometric Approach to Determination of Chondroitin/Dermatan Sulfate Oligosaccharide Glycoforms. Glycobiology 2006, 16, 502-513.

13. Hitchcock, A. M.; Costello, C. E.; Zaia, J. Glycoform Quantification of Chondroitin/Dermatan Sulfate Using a Liquid Chromatography-tandem Mass Spectrometry Platform. Biochemistry 2006, 45, 2350-2361.

14. Saad O. M.; Leary, J. A. Heparin Sequencing Using Enzymatic Digestion and ESI-MS ${ }^{\mathrm{n}}$ with HOST: A Heparin/HS Oligosaccharide Sequencing Tool. Anal. Chem. 2005, 77, 5902-5911.

15. Munoz, E.; Xu, D.; Kemp, M.; Zhang, F.; Liu, J.; Linhardt, R. J. Affinity, Kinetic, and Structural Study of the Interaction of 3-O-Sulfotransferase Isoform 1 with Heparan Sulfate. Biochemistry 2006, 45, 5122-5128.

16. McClellan, J. E.; Costello, C. E.; O'Connor, P. B.; Zaia, J. Influence of Charge State on Product Ion Mass Spectra and the Determination of 4S/6S Sulfation Sequence of Chondroitin Sulfate Oligosaccharides. Anal. Chem. 2002, 74, 3760-3771.

17. Zaia, J.; McClellan, J. E.; Costello, C. E. Tandem Mass Spectrometric Determination of the 4S/6S Sulfation Sequence in Chondroitin Sulfate Oligosaccharides. Anal. Chem. 2001, 73, 6030-6039.

18. Mikami, T.; Mizumoto, S.; Kago, N.; Kitagawa, H.; Sugahara, K. Specificities of Three Distinct Human Chondroitin/Dermatan N-Acetylgalactosamine 4-O-Sulfotransferases Demonstrated Using Partially Desulfated Dermatan Sulfate as an Acceptor: Implication of Differential Roles in Dermatan Sulfate Biosynthesis. J. Biol. Chem. 2003, 278, 3615-3627

19. Zamfir, A.; Seidler, D. G.; Schonherr, E.; Kresse, H.; Peter-Katalinić, J. On-Line Sheathless Capillary Electrophoresis/Nanoelectrospray Ionization-Tandem Mass Spectrometry for the Analysis of Glycosaminoglycan Oligosaccharides. Electrophoresis 2004, 13, 2010-2016.

20. Zamfir, A.; Peter-Katalinić, J. Capillary Electrophoresis-Mass Spectrometry for Glycoscreening in Biomedical Research. Electrophoresis 2004, 13, 1949-1963.

21. Zaia, J. Mass Spectrometry of Oligosaccharides. Mass Spectrom. Rev. 2004, 23, 161-227.

22. Gunay, N. S.; Linhardt, R. J. Capillary Electrophoretic Separation of Heparin Oligosaccharides Under Conditions Amenable to Mass Spectrometric Detection. J. Chromatogr. A 2003, 1014, 225-233.

23. Liu, J.; Shriver, Z.; Pope, R. M.; Thorp, S. C.; Duncan, M. B.; Copeland, R. J.; Raska, C. S.; Yoshida, K.; Eisenberg, R. J.; Cohen, G.; Linhardt, R. J.; Sasisekharan, R. Characterization of a Heparan Sulfate Octasaccharide that Binds to Herpes simplex Virus Type 1 Glycoprotein D. J. Biol. Chem. 2002, 277, 33456-33467.

24. Yang, H. O.; Gunay, N. S.; Toida, T.; Kuberan, B.; Yu, G.; Kim, Y. S.; Linhardt, R. J. Preparation and Structural Determination of Dermatan Sulfate-Derived Oligosaccharides. Glycobiology 2000, 10, 1033-1039.

25. Henriksen, J.; Roepstorff, P.; Ringborg, L. H. Ion-Pairing Reverse-Phase Chromatography/Mass Spectrometry of Heparin. Carbohydr. Res. 2006, $341,382-387$.

26. Henriksen, J.; Ringborg, L. H.; Roepstorrf, P. On-Line Size-Exclusion Chromatography/Mass Spectrometry of Low Molecular Mass Heparin. J. Mass Spectrom. 2004, 39, 1305-1312. 
27. Marshall, A. G.; Hendrickson, C. L.; Jackson, G. S. Fourier Transform Ion Cyclotron Resonance Mass Spectrometry: A primer. Mass Spectrom. Rev. 1998, 17, 1-35.

28. Hughey, C. A.; Rodgers, R. P.; Marshall, A. G. Resolution of 11,000 Compositionally Distinct Components in a Single Electrospray Ionization Fourier Transform Ion Cyclotron Resonance Mass Spectrum of Crude Oil. Anal. Chem. 2002, 74, 4145-4149.

29. Bergquist, J.; Palmblad, M.; Wetterhall, M.; Håkansson, P.; Markides, K. E. Peptide Mapping of Proteins in Human Body Fluids Using Electrospray Ionization Fourier Transform Ion Cyclotron Resonance Mass Spectrometry. Mass Spectrom. Rev. 2002, 21, 2-15.

30. Vakhrushev, S. Y.; Mormann, M.; Peter-Katalinić, J. Identification of Glycoconjugates in the Urine of a Patient with Congenital Disorder of Glycosylation by High-Resolution Mass Spectrometry. Proteomics 2006, 6, 983-992.
31. Aguilan, J. T.; Dayrit, F. M.; Zhang, J.; Niñonuevo, M. R.; Lebrilla, C. B. Structural Analysis of $\kappa$-Carrageenan Sulfated Oligosaccharides by Positive Mode Nano-ESI-FTICR-MS and MS/MS by SORI-CID. J. Am. Soc. Mass Spectrom. 2006, 17, 96-103.

32. Sweeney, M. D.; Yua, Y; Leary, J. A. Effects of Sulfate Position on Heparin Octasaccharide Binding to CCL2 Examined by Tandem Mass Spectrometry. J. Am. Soc. Mass Spectrom. 2006, 17, 1114-1119.

33. Patrie, S. M.; Charlebois, J. P.; Whipple, D.; Kelleher, N. L.; Hendrickson, C. L.; Quinn, J. P.; Marshall, A. G.; Mukhopadhyay, B. Construction of a Hybrid Quadrupole/Fourier Transform Ion Cyclotron Resonance Mass Spectrometer for Versatile MS/MS Above $10 \mathrm{kDa}$. J. Am. Soc. Mass Spectrom. 2004, 15, 1099-1108.

34. Wong, R. L.; Amster, I. J. Combining Low and High Mass Ion Accumulation for Enhancing Shotgun Proteome Analysis by Accurate Mass Measurement. J. Am. Soc. Mass Spectrom. 2006, 17, 96-103. 